\title{
New noncommutative gauge theories
}

\author{
L. Bonora* \\ SISSA/ISAS, Trieste, Italy, via Beirut 2-4, 34013 \\ E-mail: bonora@sissa.it
}

ABSTRACT: I present a generalization of noncommutative gauge theories to the case of orthogonal and symplectic groups. These theories represent effective theories of orientifold projections of type IIA string theory at low energy.

\section{Introduction}

It is well-known that D-branes introduce a modification of our idea of space-time. For example, the low energy configuration of many parallel $\mathrm{D}$ branes may be represented by SYM theories, [1], in which matter fields are hermitean matrices, say $X^{i}$. The difference between the diagonal entries of $X^{i}$ are interpreted as distances between branes, and, when the distances are large they are the only effective parameters. However at small distances new parameters (the nondiagonal entries of $X^{i}$, which are interpreted as open string coordinates stretched between branes) become relevant, so that actually the usual spacetime coordinates are replaced by matrices (with ensuing noncommutativity).

The above picture is very suggestive and physical, but it is not very effective from the point of view of space-time noncommutativity. It has been realized recently that this noncommutativity surfaces in a very effective and manageable way if in D-branes configurations as above we switch one a constant NSNS B-field. The point is that the components of a constant B-field which are parallel to a $D p$-brane can not be gauged away $[2,3]$ and the worldvolume of these branes become noncommutative $[2,3,4]$. In addition, by computing open string states scattering amplitudes and extracting the massless poles contributions, one can show that the low energy effective theory describing the system is the non-

*Work done in collaboration with M.M.SheikhJabbari, M.Schnabl, A.Tomasiello commutative $U(n)(N C U(n))$ theory $[5,6]$. This theory is obtained by replacing the usual products of fields by the star (Moyal) product [7].

One of the questions that arise immediately is whether $N C U(n)$ is the only noncommutative gauge field theory that makes sense. At first sight, trying to define a noncommutative gauge theory corresponding to subgroup of $U(n)$ does not look very promising: the product of two gauge transformations valued in a Lie subalgebra of $u(n)$ is not valued in the same Lie subalgebra. However the question admits in some sense a positive answer [8]. It is indeed possible to find consistent noncommutative extension for gauge theories corresponding to certain subgroups of $U(n)$. The main point is that we can define gauge transformations that close to form a subgroup of the group of $N C U(n)$ gauge transformations even though the corresponding gauge potentials and gauge transformations are not valued in a classical Lie subalgebra of the unitary Lie algebra $\mathfrak{u}(n)$.

The above question is given an answer in two different ways, one relying on purely gauge field theory considerations and the other on string theory. From the gauge theory point of view, it is shown that it is possible to impose constraints on the gauge potentials and the gauge transformation so that when the deformation parameter vanishes we recover the ordinary orthogonal and symplectic gauge theories. From the string theory point of view we construct an orientifold projection of a brane configuration which gives rise to the same amplitudes as the noncommutative 
orthogonal or symplectic gauge field theories in the $\alpha^{\prime} \rightarrow 0$ limit.

\section{Noncommutative $S O(n)$ and $S p(n)$ gauge theories}

We work in $\mathbb{R}^{d}$ and use the Moyal bracket

$$
(f * g)(x) \equiv f(x) e^{\frac{i}{2} \theta^{\mu \nu} \overleftarrow{\partial}_{\mu} \vec{\partial}_{\nu}} g(x)
$$

for functions in $\mathbb{R}^{d}$. We define the algebra $\mathcal{A}_{\theta}$ as the vector space $C^{\infty}\left(\mathbb{R}^{d}\right)$ endowed with this product.

The difficulty of defining a noncommutative gauge theory based on a subgroup of $S U(n)$ is related to the fact that the Moyal product of two gauge transformations valued in a proper subalgebra of $\mathfrak{u}(n)$, is in general not valued in the same subalgebra. However, in order to define a gauge theory, it is enough to be able to define a Lie algebra of gauge transformations. And this is possible notwithstanding this difficulty. Let us see how this may work.

To start with, we will work in a setting in which $\theta$ has to be thought of as a parameter. Accordingly, we will consider $\mathcal{A}_{\theta}$ as an algebra of power series in $\theta$. This algebra is endowed with an anti-automorphism $r$ defined by

$$
(.)^{r}: f(x, \theta) \mapsto f^{r}(x, \theta) \equiv f(x,-\theta) .
$$

This map reduces to the identity on the generators $x^{\mu}$ and reverses the order in the product: $\left(x_{1}^{\mu} * \ldots * x_{n}^{\mu}\right)^{r}=\left(x_{n}^{\mu}\right)^{r} * \ldots *\left(x_{1}^{\mu}\right)^{r}$.

First of all, we consider our groups as subgroups of $U(n)$. In other words we keep the usual antihermiticity condition on the $u(n)$-valued connections $A$ and gauge transformations $\lambda$.

$$
\begin{aligned}
A_{i j}^{*}(x, \theta) & =-A_{j i}(x, \theta) \\
\lambda_{i j}^{*}(x, \theta) & =-\lambda_{j i}(x, \theta) .
\end{aligned}
$$

By convention, we use Greek letters for spacetime indices and $i$ and $j$ for matrix (group) indices.

To define $N C S O(n)$ gauge theories we select gauge connections and transformations satisfying the following constraints:

$$
\begin{aligned}
A_{i j}^{r}(x, \theta) & =-A_{j i}(x, \theta) \\
\lambda_{i j}^{r}(x, \theta) & =-\lambda_{j i}(x, \theta)
\end{aligned}
$$

It is easy to see that these constraints are preserved by gauge transformations. One can see it componentwise. Alternatively, rewrite (2.4) in the concise form $A=-\left(A^{t}\right)^{r}$ and $\lambda=-\left(\lambda^{t}\right)^{r}$, by using the matrix transposition $t$. Define $\left((.)^{t}\right)^{r} \equiv$ $(.)^{r t}$. The proof is now formally similar to the usual one for $U(n)$, replacing $\dagger$ with $r t:(\lambda * A-$ $A * \lambda)^{r t}=A^{r t} * \lambda^{r t}-\lambda^{r t} * A^{r t}=-(\lambda * A-A * \lambda)$.

We anticipated above that due to (2.4), connections and gauge parameters do not turn out to be $s o(n)$-valued. Nevertheless (2.4) introduces restrictions on the matrix functions $A_{i j}$. To see this, let us write (2.4) more explicitly

$$
\begin{aligned}
A_{i j}(x, \theta) & =-A_{j i}(x,-\theta) \\
\lambda_{i j}(x, \theta) & =-\lambda_{j i}(x,-\theta)
\end{aligned}
$$

Inserting a power expansion in $\theta$ for $A$

$$
A^{\mu}(x, \theta)=A_{0}^{\mu}(x)+i \theta_{\nu \rho} A_{1}^{\mu \nu \rho}(x)+\ldots,
$$

we see that (2.4) implies that $A_{0}, A_{2}, \ldots$ are antisymmetric and $A_{1}, A_{3} \ldots$ symmetric. The hermiticity condition (2.3) imposes that all the coefficients $A_{0}, A_{1}, \ldots$ be real. The same conclusions hold for the power expansion of $\lambda$.

Up to now, $A_{0}, A_{1}, \ldots$ are unrestricted, except for the just mentioned constraint. However, if we want to make connection with string theory, $A_{1}, A_{2}, \ldots$ should not introduce new degrees of freedom, but be functionally dependent on $A_{0}$. In practice we will regard them as given by the Seiberg-Witten map [7]:

$A^{\mu}\left(A_{0}\right)=A_{0}^{\mu}-\frac{i}{4} \theta^{\nu \rho}\left\{A_{0 \nu}, \partial_{\rho} A_{0}^{\mu}+F_{0 \rho}{ }^{\mu}\right\}+\mathcal{O}\left(\theta^{2}\right) ;$

(the presence of $i$ is due the fact that Seiberg and Witten use hermitean connections rather than anti-hermitean ones, as we do). This is indeed consistent: the term linear in $\theta$ is symmetric if the constant part is antisymmetric. In fact, one can also see that the next term is antisymmetric, and so on; so we have complete accord with (2.6).

To define a Yang-Mills $N C S O(n)$ theory, let $A=A(x, \theta)$ satisfy the constraint (2.4). The action is the usual one

$$
S=-\frac{1}{4} \int d^{d} x F_{i j}^{\mu \nu} F_{j i \mu \nu}
$$


where $F$ is defined as

$$
F_{\mu \nu}=\partial_{[\mu} A_{\nu]}+A_{\mu} * A_{\nu}-A_{\nu} * A_{\mu} .
$$

The action (2.8) is naturally gauge invariant under $N C S O(n)$ and positive. It reduces to the usual one for $S O(n)$ in the $\theta=0$ case.

It is rather straightforward to introduce matter fields in this context in a coherent way. For example, suppose we want to introduce fermions in the adjoint representation. We can define a generalization of the SW map to such fields.

Let $\psi_{0}$ be an ordinary (commutative) spinor in the adjoint representation, which therefore transforms as follows under an ordinary gauge transformation

$$
\delta_{\lambda_{0}} \psi_{0}=\left[\psi_{0}, \lambda_{0}\right]
$$

and let us postulate the following noncommutative gauge transformation for the corresponding noncommutative field:

$$
\delta_{\lambda} \psi=\psi * \lambda-\lambda * \psi
$$

where $\lambda=\lambda_{0}+\lambda^{\prime}\left(\lambda_{0}, A_{0}\right), A=A_{0}+A^{\prime}\left(A_{0}\right)$ and $\psi=\psi_{0}+\psi^{\prime}\left(\psi_{0}, A_{0}\right)$; the primed fields are first order in $\theta$. We want to find a function $\psi\left(\psi_{0}, A_{0}\right)$ which transform as (2.11) when the corresponding $\psi_{0}$ transform as $(2.10)$. This amounts to satisfying the equation

$$
\psi\left(\psi_{0}, A_{0}\right)+\delta_{\lambda} \psi\left(\psi_{0}, A_{0}\right)=\psi\left(\psi_{0}+\delta_{\lambda_{0}} \psi_{0}, A_{0}+\delta_{\lambda_{0}} A_{0}\right)
$$

The solution to first order in $\theta$ is

$$
\begin{aligned}
& \psi\left(\psi_{0}, A_{0}\right)=\psi_{0}-\frac{i}{2} \theta^{\mu \nu} \psi_{1 \mu \nu}+\mathcal{O}\left(\theta^{2}\right) \\
& \psi_{1 \mu \nu}=\left\{A_{0 \mu}, \partial_{\nu} \psi_{0}\right\}+\frac{1}{2}\left\{\left[\psi_{0}, A_{0 \mu}\right], A_{0 \nu}\right\}
\end{aligned}
$$

It is easy to see that the noncommutative orthogonal constraint

$$
\psi^{r t}=-\psi
$$

is consistent with this map. Therefore they form a representation of $N C S O(n)$. In a similar way one can introduce also the fundamental representation. The action terms containing these matter fields are the usual ones with ordinary product replaced by the noncommutative one.

\section{A string theory origin.}

We want now to derive the gauge theory we described above from a brane configuration in string theory in the limit $\alpha^{\prime} \rightarrow 0$.

In the commutative case, gauge theories with orthogonal or symplectic groups are realized as low energy effective actions of branes on orientifold planes in type I theories. Since noncommutativity is achieved by a non zero $B$ field, and this vanishes on the orientifold plane, one may deem this search hopeless. However the question is more subtle.

For the sake of definiteness, let us consider type IA theory. This theory can be obtained in two ways: as T-dual of type IB , or as an orientifold of type IIA theory. In the second way, it is from the very beginning a 10d theory if the initial IIA is; in the first, it is of course compactified in at least one direction, and one can make contact with the other approach by taking this radius to infinity. Either way, we obtain a 10D theory.

The symmetry of IIA theory which defines the relevant orientifold is given by $P_{9} \cdot \Omega$, where $P_{9}: x^{9} \rightarrow-x^{9}$ is a spacetime reflection and $\Omega$ : $\sigma \rightarrow \pi-\sigma$ is the worldsheet parity. So, the orientifold plane is an eight-plane located at $x^{9}=$ 0 . Physics in the $x^{9}>0$ region is locally the same as the IIA one. However, strings always have an image on the other side, and as a consequence any spacetime field $\phi$ is reflected as

$$
\phi\left(x^{1}, \ldots, x^{8}, x^{9}\right)= \pm \phi\left(x^{1}, \ldots, x^{8},-x^{9}\right),
$$

the sign being determined by the $\Omega$ parity. So, in particular, the RR charges of image branes have a relative \pm according to their dimensionality. To obtain the gauge groups we are looking for, namely $S O$ and $S p$ groups, we have to put branes and their mirrors on the orientifold plane; so, as far as we are concerned only branes whose mirrors have the same RR charge survive - the others meet their antibranes and annihilate. The surviving ones are 0,4 , and 8-branes; the gauge group on them is $S O, S p$ and $S O$ respectively.

In this configuration of branes stuck on the orientifold plane, let us now analyze the consequence of a background $B$ field. Since we are really interested in its components parallel to the orientifold, we set $B_{\mu 9}=0, \mu=1 \ldots 8$. As 
for the remaining components, bearing in mind that the $B$ field is odd under worldsheet parity [9] from $(3.1)$ we see that $B_{\mu \nu}\left(x^{1}, \ldots, x^{8}, x^{9}\right)=$ $-B_{\mu \nu}\left(x^{1}, \ldots, x^{8},-x^{9}\right)$. So, we will consider a configuration $B_{\mu \nu}=b_{\mu \nu} f\left(x^{9}\right)$, where $f$ is odd in $x^{9}$. It is true that the $B$ field is zero on the orientifold, but strings which end on the branes can stretch also outside, and the usual statement that their interaction with $B$ is a boundary term is, in general, true only when $B$ is constant. The interaction term equals ( $\Sigma$ is the worldsheet of the open string)

$$
\begin{aligned}
& \int_{\Sigma} B_{\mu \nu} d x^{\mu} \wedge d x^{\nu} \\
& =\int_{\Sigma} d\left(B_{\mu \nu} x^{\mu} d x^{\nu}\right)-\int_{\Sigma} d B_{\mu \nu} \wedge x^{\mu} d x^{\nu} \\
& =\int_{\partial \Sigma} B_{\mu \nu} x^{\mu} d x^{\nu}-\int_{\Sigma} \partial_{\rho} B_{\mu \nu} d x^{\rho} \wedge x^{\mu} d x^{\nu}
\end{aligned}
$$

In the usual $B=$ constant case, the first term of the final expression is the boundary term which is responsible for noncommutativity, while the second vanishes. In the present case, the situation is different: the first term is zero, due to the vanishing of the $B$ field on the orientifold plane (branes are on the orientifold, so $\partial \Sigma \subset O 8$ ), but the second is

$$
\int_{\Sigma} \partial_{9} f\left(x^{9}\right) b_{\mu \nu} x^{\mu} d x^{\nu} \wedge d x^{9} .
$$

We choose $f$ to be the step function $\epsilon\left(x^{9}\right)$, which is in fact the easiest field configuration one can think of in this case. The factor $\partial_{9} f\left(x^{9}\right)$ becomes a $\delta\left(x^{9}\right)$, and this implies the integral concentrates on the orientifold $\left\{x^{9}=0\right\}$ :

$$
\int_{\Sigma \cap O 8} b_{\mu \nu} x^{\mu} d x^{\nu} .
$$

Now, as $\Sigma \cap O 8 \supset \partial \Sigma$, this provides a boundary term which has exactly the form of the one which usually accounts for noncommutativity.

That a system of $D p$-branes on top of an orientifold plane $(O p$-plane with $p \leq 8)$ in the presence of a step function-like $B$ field is a stable system, can be seen also with an argument based on supersymmetry, see [8].

One can now compute correlation functions of strings attached to the branes. If we specialize to gauge bosons, the corresponding vertex operator in the -1 picture is $V(z)=\xi^{i j} \cdot(\psi+\bar{\psi}) e^{i k x}$, where $\xi^{r t}=-\xi$. The three point functions, for instance is

$$
\begin{aligned}
& \operatorname{Tr}\left\{\xi_{1} \cdot p_{2} \xi_{2} \cdot \xi_{3}+\xi_{2} \cdot p_{3} \xi_{3} \cdot \xi_{1}+\xi_{3} \cdot p_{1} \xi_{1} \cdot \xi_{2}\right\} \\
& \cdot e^{-\frac{i}{2} p_{\mu}^{1} \theta^{\mu \nu} p_{\nu}^{2}}+(1 \leftrightarrow 2)
\end{aligned}
$$

where inner products are understood with respect to the open string metric. This is the same amplitude one finds starting from a noncommutative gauge theory, but with the additional constraint $\xi=-\xi^{r t}$; thus it coincides with the field theory we have suggested.

Acknowledgements. This work was supported by the EC TMR Programme, grant FMRXCT96-0012.

\section{References}

[1] E. Witten, Bound States of Strings and p-branes, Nucl. Phys. B 460 (1996) 335, hep-th/9510135.

[2] Y.-K. E. Cheung, M. Krogh, Noncommutative Geometry From 0-Branes in a Background B Field, Nucl. Phys. B 528 (1998) 185, hep-th/9803031.

F. Ardalan, H. Arfaei, M. M. Sheikh-Jabbari, Mixed Branes and M(atrix) Theory on Noncommutative Torus, hep-th/9803067.

F. Ardalan, H. Arfaei, M. M. Sheikh-Jabbari, Noncommutative Geometry form Strings and Branes, J. High Energy Phys. 02 (1999) 016, hep-th/9810072.

[3] C-S. Chu and P-M. Ho, Noncommutative Open Strings and D-branes, Nucl. Phys. B 550 (1999) 151, hep-th/9812219.

[4] F. Ardalan, H. Arfaei, M. M. Sheikh-Jabbari, Dirac Quantization of Open String and Noncommutativity in Branes, Nucl. Phys. B 576 (2000) 578, hep-th/9906161.

C.-S. Chu, P.-M. Ho, Constrained Quantization of Open Strings in Background B and Noncommutative D-Branes, Nucl. Phys. B 568 (2000) 447, hep-th/9906192.

M. M. Sheikh-Jabbari and A. Shirzad, Boundary Conditions as Dirac Constraints, hep-th/9907055.

[5] M. R. Douglas, C. Hull, D-branes and Noncommutative Torus, J. High Energy Phys. 9802 (1998) 008, hep-th/9711165. 
[6] M. M. Sheikh-Jabbari, Super Yang-Mills Theory on Noncommutative Torus From Open Strings Interactions, Phys. Lett. B 450 (1999) 119, hep-th/9810179.

[7] N. Seiberg, E. Witten, String Theory and Noncommutative Geometry, J. High Energy Phys. 09 (1999) 032, hep-th/9908142.

[8] L. Bonora, M. Schnabl, M.M. Sheikh-Jabbari, A. Tomasiello Noncommutative $S O(n)$ and $S p(n)$ gauge theories, hep-th/0006091, to appear in NPB.

[9] J. Polchinski, TASI Lectures on D-Branes , hep-th/9611050.

[10] T. Krajewski, R. Wulkenhaar Perturbative quantum gauge fields on the noncommutative torus, hep-th/9903187.

[11] A. Armoni, Comments on Perturbative Dynamics of Noncommutative Yang-Mills Theory, hep-th/0005208. 